KTH Electrical Engineering

\title{
Capacity Bounds for the Discrete Superposition Model of the Gaussian Multiple-Access Channel
}

(C2010 IEEE. Personal use of this material is permitted. However, permission to reprint/republish this material for advertising or promotional purposes or for creating new collective works for resale or redistribution to servers or lists, or to reuse any copyrighted component of this work in other works must be obtained from the IEEE.

NICOLAS SCHRAMMAR AND MIKAEL SKOGLUND

Stockholm 2010

Communication Theory Department

School of Electrical Engineering

KTH Royal Institute of Technology

IR-EE-KT 2011:001 


\title{
Capacity Bounds for the Discrete Superposition Model of the Gaussian Multiple-Access Channel
}

\author{
Nicolas Schrammar and Mikael Skoglund \\ School of Electrical Engineering and ACCESS Linnaeus Center \\ KTH Royal Institute of Technology, Stockholm, Sweden \\ Email: \{nisc, skoglund\}@kth.se
}

\begin{abstract}
Recently, it has been shown that the capacity of certain Gaussian networks can be approximated by the capacity of the corresponding network in the discrete superposition model (DSM) [1], [2]. The gap between the capacities is an additive constant only depending on the number of nodes in the network. Hence, the capacity in the DSM is a good approximation in the high SNR regime. Finding this capacity involves optimizing over a finite set of coding strategies. However, the problem space grows with both the number of nodes and with SNR, rendering the optimization infeasible.

In this paper we find upper and lower bounds on the capacity in the DSM. We start with the point-to-point channel, and we extend our strategy to the multiple-access channel. We show that the gap between our bounds is at most an additive constant independent of the channel gains. Hence, combining our results with the results of [1], [2], we find closed form bounds on the Gaussian capacity to within an additive constant.
\end{abstract}

\section{INTRODUCTION}

Finding the capacity of wireless networks is a challenging problem. The capacity of the point-to-point channel found by Shannon in 1948 [3] is a valuable figure for both research and application design, as it provides a fundamental bound for the transmission rate over the wireless medium. Finding a similar figure for wireless networks by extending Shannon's ideas turned out to be a problem of rapidly increasing difficulty. Even the capacity of small networks like the three-node relay channel is still unknown.

Recently, an alternative model, the linear finite-field deterministic model, has been proposed by Avestimehr et al. [4]. The aim was to find the capacity in the deterministic model and use it as an approximation to the capacity of the Gaussian model. However, the connection between the capacities could be established only for certain examples.

A different deterministic model, the discrete superposition model (DSM), was proposed by Anand and Kumar in [1]. It has some similarity to the truncated deterministic model proposed by Avestimehr et al. in [5]. For the DSM the authors showed that the gap between the capacity of the network in the DSM and the Gaussian model is an additive constant dependent only on the number of nodes. This result holds for relay networks with a single source and a single destination [1] and for the $K$-user interference channel [2]. An approximation within an additive constant is particularly interesting in the regime of high signal-to-noise ratio (SNR). In this case the gap becomes negligible.
The results of Anand and Kumar are the first step to approximate the capacity of a Gaussian network. The second step, that has not been addressed by the authors, is to find the capacity in the DSM. In the DSM the set of channel inputs is finite; thus, we could argue that finding this capacity is just a combinatorial problem. This is true, though, to find the capacity, we have to optimize over all possible combinations of transmit symbols. The complexity of this optimization increases dramatically, since the number of transmit symbols increases with SNR. ${ }^{1}$

In order to take the second step, this paper provides closed form bounds on the capacity in the DSM. We show that the distance between the bounds is an additive constant. Provided that there is a constant gap between the Gaussian capacity and the capacity in the DSM, our bounds are at the same time bounds on the Gaussian capacity to within a constant gap.

After introducing the DSM in Sec. II, we start by bounding the capacity of the point-to-point channel in Sec. III to illustrate our strategy. We then study the capacity of the twouser multiple-access channel (MAC), and we extend our results to the general $K$-user MAC in Sec. IV, before we conclude the paper in Sec. V.

\section{Channel Models}

\section{A. Gaussian Channel Model}

The received signal in the Gaussian model of the $K$-user MAC is

$$
y=\sum_{k=1}^{K} h_{k} x_{k}+z,
$$

where $h_{k}, x_{k}$ and $z$ denote the gain of the channel from transmitter $k$ to the receiver, the signal of transmitter $k$ and the noise at the receiver, respectively. The average transmit power is constrained to $\mathbb{E}\left|x_{k}\right|^{2} \leq 1$. The noise $z \sim \mathcal{C N}(0,1)$ is white Gaussian with unit power. Thus, the channel is completely described by the channel gains $h_{k}$.

\footnotetext{
${ }^{1}$ Consider the simple two-user multiple-access channel (MAC) at moderate SNR of $40 \mathrm{~dB}$. In this case the number of different transmit symbols is $N=2^{12}$. To share the channel, each of the transmitters chooses $k$ symbols, say $k=2^{10}$. There are $N$ over $k$ possibilities for this choice, more than $10^{616}$ in our example. We show that the problem of finding the optimal subset of transmit symbols can be expressed as the problem of finding the largest clique in a graph of size $N$. However, this is another combinatorial problem and thus does not improve the scaling of the complexity.
} 


\section{B. Discrete Superposition Model}

Define the rounding operation

$$
[a]=\operatorname{sgn}\left(a_{\mathrm{R}}\right)\left\lfloor\left|a_{\mathrm{R}}\right|\right\rfloor+j \operatorname{sgn}\left(a_{\mathrm{I}}\right)\left\lfloor\left|a_{\mathrm{I}}\right|\right\rfloor,
$$

where the index R or I denotes the real or imaginary part, respectively. The MAC in the DSM is modeled as

$$
y=\sum_{k=1}^{K}\left[\left[h_{k}\right] x_{k}\right] .
$$

The transmitted signal $x_{k}$ is scaled by a rounded version $\left[h_{k}\right]$ of the channel gain. The result is rounded again and added to the signal components received from other transmitters. Note that this is a standard addition in $\mathbb{Z}+j \mathbb{Z}$ as opposed to the linear finite-field deterministic model in [4]. The DSM is deterministic because it lacks a noise term. Essentially, the Gaussian noise is replaced by quantization noise induced by the rounding operation.

Additionally, the transmitted signals $x_{k}$ are constrained to having discrete values as follows. Let

$$
n=\max _{k} \max \left\{\left\lfloor\log \left|h_{k \mathrm{R}}\right|\right\rfloor,\left\lfloor\log \left|h_{k \mathrm{I}}\right|\right\rfloor\right\} .
$$

Note that all logarithms in this papers are to the base 2. The complex parts $x_{k \mathrm{R}}$ and $x_{k \mathrm{I}}$ take values out of the set

$$
\left\{0, \frac{2^{-n}}{\sqrt{2}}, \ldots, \frac{1-2^{-n}}{\sqrt{2}}\right\}
$$

of $2^{n}$ equally spaced points. Hence, there are $2^{2 n}$ possible transmit symbols at each transmitter.

\section{Capacity of Point-to-Point Channel}

For the purpose of illustration we consider the point-to-point channel first. It will turn out that many of the tools developed here can be used for finding the capacity of the MAC. In the DSM the point-to-point channel is modeled as

$$
y=[[h] x] \text {. }
$$

Due to the rounding operation, $y$ lies in $\mathbb{Z}+j \mathbb{Z}$. Furthermore, the region in which $y$ lies is bounded because the region of $x$ is bounded. Let $\mathcal{Y}$ be the set of possible values for $y$ and let $m=|\mathcal{Y}|$ be its cardinality.

Lemma 1: The capacity of the point-to-point channel in the DSM is

$$
C=\log m .
$$

It can be achieved by code words of length one.

Proof: The capacity is constrained by both the number of transmit symbols $2^{2 n}$ and the number of receive symbols $m$. Clearly,

$$
C \leq \min (2 n, \log m)=\log m,
$$

because $2^{2 n}$ transmit symbols can result in at most $2^{2 n}$ receive symbols. The transmitter uses only those $m$ transmit symbols that result in distinct receive symbols. Using this set with uniform probability achieves the rate $\log m$.

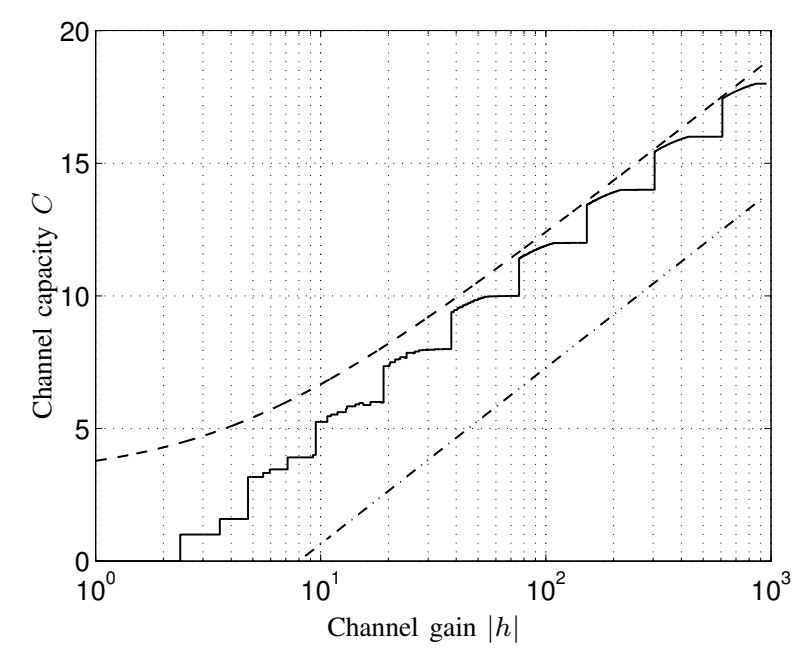

Fig. 1. Capacity of point-to-point channel with upper and lower bound due to Lem. 2 and Lem. 3, respectively. The channel gain is $h=|h| e^{j}$.

It is not necessary to use long code words because there is no randomness in the channel that has to be averaged.

The number $m$ of receive symbols can be found by simulation. Each of the possible transmit symbols $x$ is passed through the channel (4), and the number of distinct receive symbols is counted. The resulting capacity is depicted in Fig. 1 with a solid line. The function has characteristic steps caused by the increasing number $2^{2 n}$ of transmit symbols, where $n \in \mathbb{N}$. On each step there is a slope followed by a flat part. In the flat part all $2^{2 n}$ receive symbols are distinct. The capacity is $C=2 n$, and it is limited by the number of transmit symbols. In the slope part some of the receive symbols merge. The capacity is limited by the number of receive symbols. Note that the capacity is slightly dependent on the angle of the channel gain. However, we will find bounds that are independent of the angle.

The shape of the graph suggests that finding a closed form expression for the capacity is difficult. To characterize the capacity we find upper and lower bounds that have a distance of a constant number of bits. Recall that the capacities of the DSM and the Gaussian model are within a constant number of bits [1]. This means that the capacity of the Gaussian model is within a constant gap to the bounds that we find. Such a constant gap approximation can be a useful tool to characterize the Gaussian capacity in the high SNR regime.

\section{A. Bounds on the Capacity of Point-to-Point Channel}

In the following we find an upper bound on the capacity of the point-to-point channel in the DSM as well as an achievable rate. We show that those two bounds are within a constant gap of 6 bits.

Lemma 2: The capacity of the point-to-point channel can be upper-bounded as

$$
C \leq \log \left(\frac{|h|^{2}}{2}+3 \sqrt{2}|h|+9\right)
$$


Proof: We investigate how many different values $y$ can take. Consider that

$$
x_{\mathrm{R}} \leq \frac{1-2^{-n}}{\sqrt{2}}<\frac{1}{\sqrt{2}} ;
$$

that is, $x$ lives in a square with side length $1 / \sqrt{2}$. Therefore $y$ lives in a square as well. The corresponding side length is

$$
\left|y\left(x=\frac{1}{\sqrt{2}}\right)\right|=\left|\left[\frac{[h]}{\sqrt{2}}\right]\right| \leq\left|\frac{[h]}{\sqrt{2}}\right| \leq \frac{|h|}{\sqrt{2}} .
$$

Moreover, the rouding operation in (4) rounds values in a square of unit side length to the same value. Hence we can upper bound the number $m$ of different values of $y$ by the number of squares of unit side length covered by a square of side length $|h| / \sqrt{2}$,

$$
\begin{aligned}
m & \leq\left\lceil\frac{|h|}{\sqrt{2}}\right]^{2}+4\left\lceil\frac{|h|}{\sqrt{2}}\right\rceil+4 \\
& \leq\left(\frac{|h|}{\sqrt{2}}+1\right)^{2}+4\left(\frac{|h|}{\sqrt{2}}+1\right)+4
\end{aligned}
$$

Next, we derive an achievable rate. The main idea is to reduce the number of transmit symbols in order to avoid merging of symbols at the receiver.

Lemma 3: Over the point-to-point channel with $|h| \geq 5$ we can achieve a rate of

$$
R=2 \log |h|-6 .
$$

Proof: Two symbols $x_{1}, x_{2}$ merge at the transmitter if $\left[[h] x_{1}\right]=\left[[h] x_{2}\right]$. Equivalently we can ask how close the two point $[h] x_{1}$ and $[h] x_{2}$ may be before the rounding operation. It is easy to see that the worst case happens around zero. If $[h] x_{1}=1+j-\varepsilon$ and $[h] x_{2}=-1-j+\varepsilon$ for a small complex valued $\varepsilon$, the two points merge at zero. Thus, if

$$
\left|[h] x_{1}-[h] x_{2}\right| \geq 2 \sqrt{2},
$$

we can avoid any merging of receive symbols.

According to (3), the distance between two transmit symbols is at least $2^{-n} / \sqrt{2}$. We can increase the distance by using only a part of the symbols. By using every second symbol in a regular fashion we can increase the distance by a factor of $\sqrt{2}$. This reduces the achievable rate by one bit. Hence, we can achieve a rate of $R=2 n-p$ with symbols that have a distance of at least

$$
\frac{2^{-n}}{\sqrt{2}}(\sqrt{2})^{p}
$$

We can bound

$$
\begin{aligned}
\left|[h] x_{1}-[h] x_{2}\right| & =|[h]|\left|x_{1}-x_{2}\right| \\
& \geq|[h]| \frac{2^{-n}}{\sqrt{2}}(\sqrt{2})^{p} \geq 2 \sqrt{2},
\end{aligned}
$$

where the last inequality is to fulfill the condition (5). This inequality yields

$$
p=[2 n+4-2 \log |[h]|] .
$$

Hence, sacrificing this number of bits avoids merging of symbols. The resulting rate is

$$
\begin{aligned}
R & =2 n-p=\lfloor 2 \log |[h]|\rfloor-4 \\
& \geq 2 \log (|h|-\sqrt{2})-5 \\
& \geq 2 \log \left(\frac{|h|}{\sqrt{2}}\right)-5=2 \log |h|-6,
\end{aligned}
$$

where we have used $|h| \geq 5$ in (6).

The bounds due to Lem. 2 and Lem. 3 are depicted in Fig. 1 . We summarize the results in the following theorem.

Theorem 1: For $|h| \geq 5$ the capacity of the point-to-point channel in the DSM can be approximated as

$$
2 \log |h|-6 \leq C \leq 2 \log |h|+1 .
$$

Proof: Consider that $|h|^{2} / 2+3 \sqrt{2}|h|+9 \leq 2|h|^{2}$ for $|h| \geq 5$ in Lem. 2 to obtain the upper bound.

\section{Capacity of Multiple-Access Channel}

In this section we first characterize the capacity region of the two-user MAC in the DSM. We then extend our results to the $K$-user MAC.

\section{A. Capacity of Two-User MAC}

The two-user MAC is modeled as

$$
y=\left[\left[h_{1}\right] x_{1}\right]+\left[\left[h_{2}\right] x_{2}\right] .
$$

We find inner and outer bounds for the capacity region, and we show that the distance of the bounds is at most a constant number of 13 bits.

Lemma 4: The capacity region of the two-user MAC is outer-bounded by

$$
\begin{aligned}
C_{1} & \leq \log m_{1}, \\
C_{2} & \leq \log m_{2}, \\
C_{1}+C_{2} & \leq \log m .
\end{aligned}
$$

Here $m=|\mathcal{Y}|$ is the cardinality of the set of receive symbols, and $m_{i}=\left|\mathcal{Y}_{i}\right|$ are the cardinalities of the sets of receive symbols in the corresponding point-to-point channel $y_{i}=\left[\left[h_{i}\right] x_{i}\right]$.

Proof: The proof is analogous to the proof of Lem. 1.

Fig. 2 depicts bounds for the case $h_{1}=9 e^{j}, h_{2}=10 e^{4 j}$. The outer bound above corresponds to the solid pentagon. The numbers $m_{1}, m_{2}$ and $m$ of distinct receive symbols have been found by simulation.

Lemma 5: The capacity region of the two-user MAC is inner-bounded by the closure of the convex hull of all rate pairs $\left(R_{1}, R_{2}\right)$ satisfying

$$
R_{1}=\log \left|\mathcal{X}_{1}\right|, \quad R_{2}=\log \left|\mathcal{X}_{2}\right|,
$$




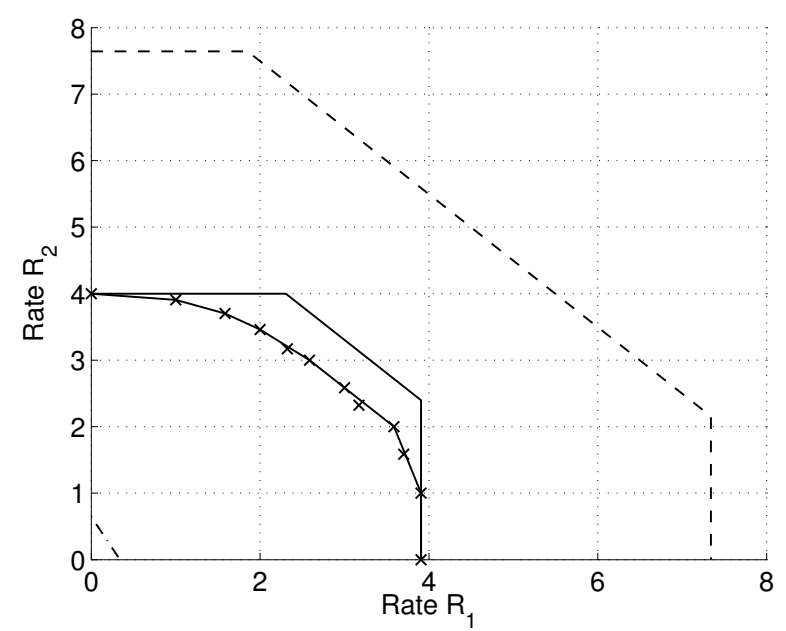

Fig. 2. Inner and outer bounds on the capacity region of the two-user MAC for $h_{1}=9 e^{j}$ and $h_{2}=10 e^{4 j}$. Solid lines are due to Lem. 4 and Lem. 5; crosses mark achievable rate pairs. The closed form bounds of Lem. 6 and Lem. 7 are depicted as dashed and dash-dotted lines, respectively.

for sets $\mathcal{X}_{1}$ and $\mathcal{X}_{2}$ of transmit symbols that result in distinct receive symbols; that is, the members of the set of receive symbols

$$
\left\{y=\left[\left[h_{1}\right] x_{1}\right]+\left[\left[h_{2}\right] x_{2}\right]: x_{1} \in \mathcal{X}_{1}, x_{2} \in \mathcal{X}_{2}\right\}
$$

are distinct. This rate region can be achieved by code words of length one.

Proof: Given the two sets $\mathcal{X}_{1}$ and $\mathcal{X}_{2}$ we can achieve the rate pair $\left(R_{1}, R_{2}\right)$ by using the symbols with uniform probability. The convex hull is achievable by using time sharing between two rate pairs.

Obtaining pairs of sets $\left(\mathcal{X}_{1}, \mathcal{X}_{2}\right)$ is a difficult process of combinatorial complexity. Although we use a graph theoretical representation of the problem ${ }^{2}$, it is impractical to find rate pairs for large channel gains. The channel gains used in the example of Fig. 2 result in $n=2$; that is, we have $2^{2 n}=$ 16 possible transmit symbols. In this example rate pairs can be found in reasonable time. The rate pairs are marked with crosses; the convex hull is depicted by a solid line.

In the next step we find outer and inner bounds that have a closed form expression in order to prove that the capacity region of the two-user MAC is within a constant gap to our bounds. It is convenient to assume without loss of generality $\left|h_{1}\right| \leq\left|h_{2}\right|$ from now on.

Lemma 6: For $\left|h_{2}\right| \geq\left|h_{1}\right| \geq 5$ the capacity region of the two-user MAC is outer-bounded by

$$
\begin{aligned}
C_{1} & \leq 2 \log \left(\left|h_{1}\right|\right)+1 \\
C_{2} & \leq 2 \log \left(\left|h_{2}\right|\right)+1, \\
C_{1}+C_{2} & \leq 2 \log \left(\left|h_{1}\right|+\left|h_{2}\right|\right)+1 .
\end{aligned}
$$

\footnotetext{
${ }^{2}$ Fix the set of transmit symbols for one transmitter. Build a graph with nodes representing the transmit symbols of the other transmitter. Connect pairs of transmit symbols that can be used so that the resulting receive symbols are disjoint. In the resulting graph search for the largest clique, the largest complete subgraph.
}

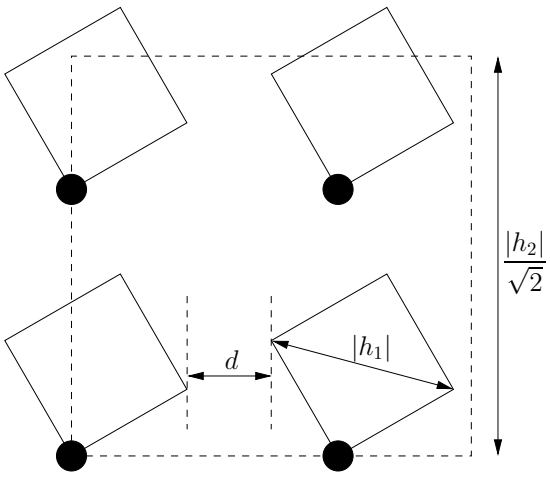

Fig. 3. Illustration for the proof of Lem. 7.

Proof: We follow the proof of Lem. 2. The signals $\left[\left[h_{1}\right] x_{1}\right]$ and $\left[\left[h_{2}\right] x_{2}\right]$ live in squares of side length $\left|h_{1}\right| / \sqrt{2}$ and $\left|h_{2}\right| / \sqrt{2}$, respectively. Hence, the sum of the signals lives in a square with side length $\left|h_{2}\right| / \sqrt{2}+\left|h_{1}\right|$. This square covers at most

$$
\begin{aligned}
m & \leq\left\lceil\frac{\left|h_{2}\right|}{\sqrt{2}}+\left|h_{1}\right|\right]^{2}+4\left\lceil\frac{\left|h_{2}\right|}{\sqrt{2}}+\left|h_{1}\right|\right]+4 \\
& \leq 2\left(\left|h_{1}\right|+\left|h_{2}\right|\right)^{2}
\end{aligned}
$$

squares of unit side length. The bounds on the individual rates are analogous to Th. 1 .

The outer bound is depicted in Fig. 2 with a dashed line.

Lemma 7: For $\left|h_{1}\right| \geq 5$ the capacity region of the two-user MAC is inner-bounded by the closure of the convex hull of the three rate pairs

$$
\begin{array}{ll}
R_{1}^{\prime}=0, & R_{2}^{\prime}=2 \log \left|h_{2}\right|-6, \\
R_{1}^{\prime \prime}=2 \log \left|h_{1}\right|-6, & R_{2}^{\prime \prime}=2 \log \left(\frac{\left|h_{2}\right|}{\left|h_{1}\right|}\right)-4, \\
R_{1}^{\prime \prime \prime}=2 \log \left|h_{1}\right|-6, & R_{2}^{\prime \prime \prime}=0 .
\end{array}
$$

Proof: The first and third rate pair are achievable due to Lem. 3. The strategy to achieve the second rate pair is the following. Transmitter 1 behaves as if transmitter 2 were not present. It transmits at a rate $R_{1}^{\prime \prime}=2 \log \left|h_{1}\right|-6$, and the received signal $\left[\left[h_{1}\right] x_{1}\right]$ occupies at most a square of side length $\left|h_{1}\right| / \sqrt{2}$. Transmitter 2 has a larger channel gain, therefore, it can use some of its transmit symbols even though transmitter 1 transmits at maximum rate. The distance of symbols used by transmitter 2 has to be large enough to avoid merging of receive symbols in the sum signal $\left[\left[h_{1}\right] x_{1}\right]+\left[\left[h_{2}\right] x_{2}\right]$.

Consider the sketch in Fig. 3. The squares occupied by transmitter 1 and 2 are depicted as solid and dashed squares respectively. Transmitter 2 uses only those symbols marked by black circles. In order to avoid merging of symbols, the shifted squares may not overlap. Hence, the distance between black dots has to be at least $\left|h_{1}\right|$ to account for all possible angles of the channel gains. 
The distance $d$ in Fig. 3 accounts for the fact that receive symbols are at discrete positions. If transmitter 2 picks symbols that have at least a distance of $\left|h_{1}\right|$, it has to pick a symbol that is a distance of $d$ further away. We can upper bound $d$ by the maximum distance of two neighboring symbols, thus,

$$
\begin{aligned}
d & \leq\left|\left[\left[h_{2}\right] x_{2}^{\prime}\right]-\left[\left[h_{2}\right] x_{2}^{\prime \prime}\right]\right| \\
& \leq\left|\left[h_{2} x_{2}^{\prime}\right]-[\underbrace{\left(h_{2}-\operatorname{sgn} h_{2 \mathrm{R}}-j \operatorname{sgn} h_{2 \mathrm{I}}\right) x_{2}^{\prime \prime}}_{\tilde{h}}]\right| \\
& \leq \mid h_{2} x_{2}^{\prime}-\left(\left(h_{2}-\operatorname{sgn} h_{2 \mathrm{R}}-j \operatorname{sgn} h_{2 \mathrm{I}}\right) x_{2}^{\prime \prime}\right. \\
& \leq\left|h_{2}\right|\left|x_{2}^{\prime}-x_{2}^{\prime \prime}\right|+2 \sqrt{2} \\
& =\frac{\left|h_{2}\right|}{\sqrt{2}} 2^{-n}+2 \sqrt{2} \\
& \leq \frac{\left|h_{2}\right|}{\sqrt{2}} \frac{2}{\max \left(\left|h_{2 \mathrm{R}}\right|,\left|h_{2 \mathrm{I}}\right|\right)}+2 \sqrt{2} \\
& \leq \frac{\left|h_{2}\right|}{\sqrt{2}} \frac{2 \sqrt{2}}{\left|h_{2}\right|}+2 \sqrt{2}=2+2 \sqrt{2} \leq 5 .
\end{aligned}
$$

The equality (10) holds because $x_{2}^{\prime}$ and $x_{2}^{\prime \prime}$ are neighboring symbols.

It remains to find a lower bound on the length of the square occupied by transmitter 2 . We find that

$$
\begin{aligned}
\left|\left[\left[h_{2}\right] x_{2, \max }\right]\right| & =\left|\left[\left[h_{2}\right] \frac{1-2^{-n}}{\sqrt{2}}\right]\right| \\
& \geq\left|\left[\left[h_{2}\right] \sqrt{2}\right]\right| \\
& \geq\left(\left|h_{2}\right|-\sqrt{2}\right) \sqrt{2}-\sqrt{2} \\
& =\sqrt{2}\left|h_{2}\right|-2-\sqrt{2} .
\end{aligned}
$$

This means that transmitter 2 can use at least

$$
\left(\frac{\sqrt{2}\left|h_{2}\right|-2-\sqrt{2}}{\left|h_{1}\right|+5}\right)^{2} \geq\left(\frac{\left|h_{2}\right|}{4\left|h_{1}\right|}\right)^{2}
$$

of its transmit symbols. Hence,

$$
R_{2}^{\prime \prime}=2 \log \left(\frac{\left|h_{2}\right|}{\left|h_{1}\right|}\right)-4
$$

is achievable.

The achievable region is depicted in Fig. 2 with a dashdotted line. However, in this example $R_{2}^{\prime \prime}=0$, and therefore, the tetragon reduces to a triangle. Consider Fig. 4 for a different example where $R_{2}^{\prime \prime}>0$.

Finally, we show that the gap between the bounds in Lem. 6 and Lem. 7 is constant and independent of the channel gains. Hence, in the high SNR region the gap is negligible.

Theorem 2: The capacity of the two-user MAC is bounded by Lem. 6 and Lem. 7 within a constant gap of 13 bits.

Proof: Consider the outer and inner bound depicted in Fig. 4 as dashed and dash-dotted line, respectively. Due to the

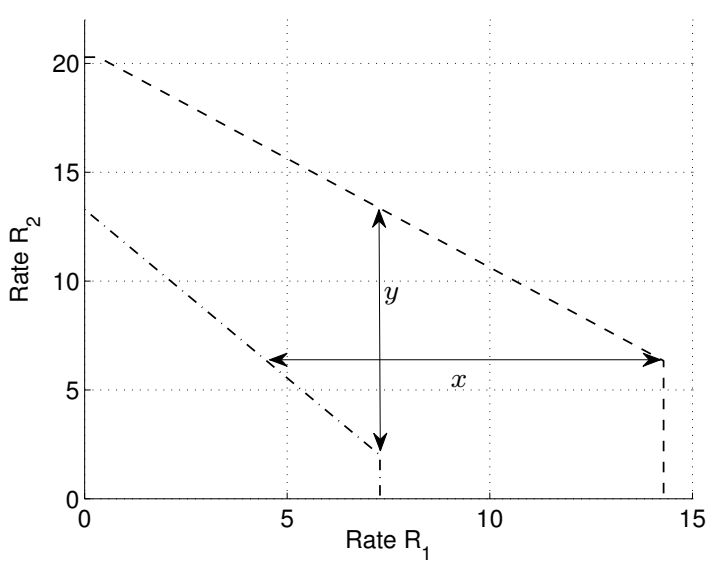

Fig. 4. Inner and outer bound on the capacity region of the two-user MAC for $h_{1}=100 e^{2 j}$ and $h_{2}=800 e^{j}$. The bounds of Lem. 6 and Lem. 7 are depicted as dashed and dash-dotted lines, respectively. The largest gaps are marked as $x$ and $y$.

different slope of the bounds on the sum-rate, the largest gaps between the bounds occur at the points marked by arrows. We upper-bound the distances $x$ and $y$.

To simplify calculations, we use a slightly looser inner bound on the capacity region. Consider $R_{2}^{\prime}=2 \log \left|h_{2}\right|-10$ in Lem. 7 that yields the inner bound

$$
\begin{aligned}
C_{1} & \geq 2 \log \left|h_{1}\right|-6, \\
C_{1}+C_{2} & \geq 2 \log \left|h_{2}\right|-10 .
\end{aligned}
$$

The arrow marked by $x$ is at the position

$$
R_{2, x}=2 \log \left(\left|h_{1}\right|+\left|h_{2}\right|\right)+1-\left(2 \log \left|h_{1}\right|+1\right) .
$$

Thus, the distance $x$ can be upper-bounded as

$$
\begin{aligned}
x & =2 \log \left|h_{1}\right|+1-\left(2 \log \left|h_{2}\right|-10-R_{2, x}\right) \\
& =2 \log \left(1+\frac{\left|h_{1}\right|}{\left|h_{2}\right|}\right)+11 \\
& \leq 2 \log 2+11=13 .
\end{aligned}
$$

To upper bound $y$ we have $R_{1, y}=2 \log \left|h_{1}\right|-6$, and hence,

$$
\begin{aligned}
y= & 2 \log \left(\left|h_{1}\right|+\left|h_{2}\right|\right)+1-R_{1, y} \\
& -\left(2 \log \left|h_{2}\right|-2 \log \left|h_{1}\right|-4\right) \\
= & 2 \log \left(1+\frac{\left|h_{1}\right|}{\left|h_{2}\right|}\right)+11 \leq 13 .
\end{aligned}
$$

\section{B. Capacity of K-User MAC}

In this part we extend our work to the $K$-user MAC (2). Again, we find an outer bound on the capacity region as well as a set of achievable rate tuples that span an achievable rate region. The gap between the bounds is a constant that depends only on the number $K$ of transmitters.

Lemma 8: For $\left|h_{k}\right| \geq 5$, the capacity region of the $K$-user MAC is bounded by a set of $2^{K}-1$ outer bounds

$$
\sum_{k \in \mathcal{S}} C_{k} \leq 2 \log \left(\sum_{k \in \mathcal{S}}\left|h_{k}\right|\right)+1, \text { for all } \mathcal{S} \subseteq\{1, \ldots, K\} \text {. }
$$


Proof: Define $\bar{k}=\arg \max _{k \in \mathcal{S}}\left|h_{k}\right|$. We extend the proof of Lem. 6 in the sense that the received signal $y$ lives in a square of side length

$$
a_{\mathcal{S}}=\frac{\left|h_{\bar{k}}\right|}{\sqrt{2}}+\sum_{k \in \mathcal{S} \backslash \bar{k}}\left|h_{k}\right| .
$$

This square covers at most a number of

$$
m_{\mathcal{S}} \leq\left\lceil a_{\mathcal{S}}\right\rceil^{2}+4\left\lceil a_{\mathcal{S}}\right\rceil+4 \leq 2 a_{\mathcal{S}}^{2} \leq 2\left(\sum_{k \in \mathcal{S}}\left|h_{k}\right|\right)^{2}
$$

squares of unit side length.

Define $\underline{k}=\arg \min _{k \in \mathcal{S}}\left|h_{k}\right|$.

Lemma 9: For $\left|h_{k}\right| \geq 5$, the capacity region of the $K$-user MAC is inner-bounded by the closure of the convex hull of the rate tuples $\left(R_{k}\right), k \in \mathcal{S}$ with

$$
\begin{aligned}
& R_{\underline{k}}=2 \log \left|h_{\underline{k}}\right|-6, \\
& R_{k}=2 \log \left(\frac{\left|h_{k}\right|}{\sum_{l \in \mathcal{S},\left|h_{l}\right| \leq\left|h_{k}\right|}\left|h_{l}\right|}\right)-4, \quad k \in \mathcal{S} \backslash \underline{k},
\end{aligned}
$$

for all $\mathcal{S} \subseteq\{1, \ldots, K\}$.

Proof: The strategy to achieve the above rate tuples is a straight forward extension of the strategy used to achieve the rates in Lem. 7. We sort the transmitters $k \in \mathcal{S}$ in the order of increasing channel gains $\left|h_{k}\right|$. The weakest transmitter transmits at rate $R_{1}=R_{\underline{k}}$. This occupies a square of side length $\left|h_{1}\right| / \sqrt{2}$ at the receiver. Hence, the second weakest transmitter can use symbols that are received at a distance of $\left|h_{1}\right|+5$. This accounts for angles of the channel gains and for the discrete set of transmit symbols. The corresponding rate of the second weakest transmitter is

$$
R_{2}=2 \log \left(\frac{\sqrt{2}\left|h_{2}\right|-2-\sqrt{2}}{\left|h_{1}\right|+5}\right) \geq 2 \log \left(\frac{\left|h_{2}\right|}{\left|h_{1}\right|}\right)-4 .
$$

Following this scheme, the third transmitter can use symbols that are received at a distance of $\left|h_{1}\right|+\left|h_{2}\right|+5$. Thus, the achievable rate is

$R_{3}=2 \log \left(\frac{\sqrt{2}\left|h_{3}\right|-2-\sqrt{2}}{\left|h_{1}\right|+\left|h_{2}\right|+5}\right) \geq 2 \log \left(\frac{\left|h_{3}\right|}{\left|h_{1}\right|+\left|h_{2}\right|}\right)-4$.

Continuing this scheme yields the expression for $R_{k}$.

It remains to show that the two bounds on the capacity are within a constant gap. We prove the following property of the rate regions.

Theorem 3: For every $\mathcal{S} \subseteq\{1, \ldots, K\}$ the distance between the achievable sum-rate and the outer bound of the capacity region is at most a constant

$$
\sum_{k \in \mathcal{S}} C_{k}-\sum_{k \in \mathcal{S}} R_{k} \leq 2 \log (K !)+4 K+3
$$

depending only on the number $K$ of transmitters.
Proof: Define $s=|\mathcal{S}|$ and sort the transmitters in the order of increasing channel gains, that is, $\left|h_{1}\right| \leq \cdots \leq\left|h_{s}\right|$. Then $R_{1}=2 \log \left|h_{1}\right|-6$, and for $k \in\{2, \ldots, s\}$

$$
\begin{aligned}
R_{k} & =2 \log \left(\frac{\left|h_{k}\right|}{\sum_{l=1}^{k-1}\left|h_{l}\right|}\right)-4 \\
& \geq 2 \log \left(\frac{\left|h_{k}\right|}{(k-1)\left|h_{k-1}\right|}\right)-4 .
\end{aligned}
$$

Summing up all the rates yields

$$
\begin{aligned}
\sum_{k=1}^{s} R_{k} & \geq 2 \log \left(\frac{\left|h_{s}\right|}{(s-1)(s-2) \times \cdots \times 2}\right)-4(s-1)-6 \\
& =2 \log \left|h_{s}\right|-2 \log ((s-1) !)-4 s-2 .
\end{aligned}
$$

Consider

$$
\sum_{k \in \mathcal{S}} C_{k} \leq 2 \log \left(\sum_{k \in \mathcal{S}}\left|h_{k}\right|\right)+1 \leq 2 \log \left(s\left|h_{s}\right|\right)+1
$$

and $K \geq s$ to finish the proof.

\section{CONCLUSION}

In this paper we provided bounds on the capacity of the DSM. The motivation of our work is the fact that the capacity of the DSM is a constant gap approximation to the Gaussian capacity in certain cases. Since finding the capacity of the DSM by exhaustive search optimization is in general infeasible, our results are important in the use of the DSM.

We studied the capacity of the point-to-point channel as well as the capacity region of the $K$-user MAC. In both cases we could show that our bounds are within a constant additive gap. This result is important. It guaranties that our bounds are at the same time bounds to the Gaussian capacity within a constant gap, provided that the Gaussian capacity and the capacity in DSM are within a constant gap.

The strategies that were used in this paper can possibly be extended to study larger networks, for which no approximation of the Gaussian capacity is known, yet.

\section{REFERENCES}

[1] M. Anand and P. R. Kumar, "On approximating Gaussian relay networks by deterministic networks," in Proc. Information Theory Workshop (ITW), Oct. 2009.

[2] _ "A digital interface for Gaussian relay and interference networks: Lifting codes from the discrete superposition model," submitted to IEEE Trans. Information Theory, May 2010, arXiv:1005.0167v1.

[3] C. E. Shannon, "A mathematical theory of communication," The Bell System Technical Journal, vol. 27, pp. 379-423,623-656, July, October 1948.

[4] S. Avestimehr, S. N. Diggavi, and D. N. C. Tse, "A deterministic approach to wireless relay networks," in Proc. Allerton Conf. on Communication, Control and Computing, Sep. 2007.

[5] — "Wireless network information flow: A deterministic approach," to appear in IEEE Trans. Information Theory, Jun. 2009, last revised Sep. 2010, arXiv:0906.5394v5. 